\title{
Mössbauer relaxation study of nonlinear excitations in pure and impure Ising-type ferromagnetic quantum chains
}

\author{
H. J. M. de Groot, L. J. de Jongh, and R. C. Thiel \\ Kamerlingh Onnes Laboratory, University of Leiden, 2300 RA Leiden, \\ The Netherlands \\ J. Reedijk \\ Gorlaeus Laboratories, University of Leiden, \\ 2300 RA Leiden, The Netherlands
}

(Received 19 March 1984)

\begin{abstract}
Nonlinear domain-wall dynamics in the quasi-one-dimensional ferromagnet $\mathrm{FeCl}_{2}$ (pyridine) 2 is studied by Mössbauer and susceptibility experiments. In particular, the influence of doping with nonmagnetic impurities is considered. In the interpretation of the experiments the predictions for classical sine-Gordon solitons are compared with those for quantum-mechanical magnon bound states in the $S=\frac{1}{2}$ discrete Ising-type ferromagnetic chain.
\end{abstract}

In recent years the application of the soliton (domain wall) concept to solid-state physics has become of increasing importance. To complement rapid theoretical developments, experimental studies using different probes are called for. In this connection, quasi-one-dimensional (1D) magnetic systems offer quite promising possibilities. ${ }^{1}$

In a previous Letter, ${ }^{2}$ we have shown that relaxation phenomena observed in the Mössbauer spectra of $\mathrm{Fe}^{2+}$ antiferromagnetic chains with Ising-type anisotropy could be unambiguously explained by the propagation of $\pi$ domain walls along the chains. In the presence of these, the fliprate $\Gamma_{\omega}$ of the electron spins becomes proportional to the product $n_{s} v_{s}$ of wall density $n_{s}$ and average wall velocity $v_{s} .3,4$ The hyperfine interaction between the nuclear and electronic spins of the $\mathrm{Fe}$ atom gives rise to highly broadened Mössbauer absorption lines as soon as $\Gamma_{\omega}$ becomes of the order of the nuclear Larmor frequency $\omega_{L}$, the broadening disappearing for $\Gamma_{\omega} \gg \omega_{L}$ or $\Gamma_{\omega}<<\omega_{L}$. Since $n_{s} v_{s} \propto \exp \left(-E_{s} / k_{B} T\right)$, where $E_{s}$ is the wall creation energy, the wall propagation should lead to a contribution to the apparent Mössbauer linewidth $(\Gamma)$ that increases exponentially as the temperature is lowered, as was experimentally verified in our previous work. In theory the broadening should reach a maximum for $\Gamma_{\omega} \approx \omega_{L}$, and decrease exponentially again at low enough temperatures, a fully magnetically split spectrum appearing for $\Gamma_{\omega}<<\omega_{L}$. This effect was not seen in the previous experiment, due to the occurrence of $3 \mathrm{D}$ magnetic order between the chains, which blocks the wall propagation and thus "switches off" the line broadening. In the present study we cover the whole relaxation process by doping the chains with very small amounts $(c<1 \%)$ of nonmagnetic $\mathrm{Cd}$ ions, which is known to reduce drastically the value of $T_{c}$. Evidently, the shorter average length of the chain segments in the doped systems may also affect the wall dynamics, ${ }^{5}$ which was an additional reason to perform this study. Furthermore, the presently investigated compound $\mathrm{FeCl}_{2} \mathrm{py}_{2}\left(\mathrm{py}=\mathrm{NC}_{5} \mathrm{H}_{5}\right)$ is a quasi 1D ferromagnetic quantum chain, ${ }^{6-8}$ as distinct from the previously investigated antiferromagnetic chains. This is of importance, since the dynamics in ferromagnetic Ising-type systems has a different origin than in the antiferromagnetic counterparts. Lastly, we confront the classical sine-Gordon (SG) descrip- tion of the nonlinear excitations with its extreme quantum analog: the $S=\frac{1}{2}$ Ising-type chain, in order to study the quantum-mechanical effects.

We have performed Mössbauer experiments on pure $\mathrm{FeCl}_{2} \mathrm{py}_{2}$ and on material doped with $\mathrm{Cd}$ concentrations $c=0.47 \%$ and $c=0.94 \%$. The pure compound is reported to be $3 \mathrm{D}$ ordered at $4.2 \mathrm{~K}$, with the ferromagnetic chains arranged in an antiferromagnetic array. ${ }^{8}$ From our own magnetic susceptibility $(\chi)$ experiments (Fig. 1), the ordering temperature is determined as $T_{c}=6.6 \pm 0.3 \mathrm{~K}$. For the doped samples, $T_{c}$ is estimated to be $T_{c}=3.0 \pm 0.5 \mathrm{~K}$ and $T_{c}=2.0 \pm 0.5 \mathrm{~K}$ for $c=0.47 \%$ and $c=0.94 \%$ (also from $\chi$ data).

The $S=\frac{1}{2}$ Ising behavior at low temperatures ( $T$ $<30 \mathrm{~K}$ ), which is due to a pseudodoublet ground state of the $\mathrm{Fe}^{2+}$ electronic spin, is confirmed by the behavior of $\chi(T)$ in Fig. 1. The data are compared with theory ${ }^{9}$ for the

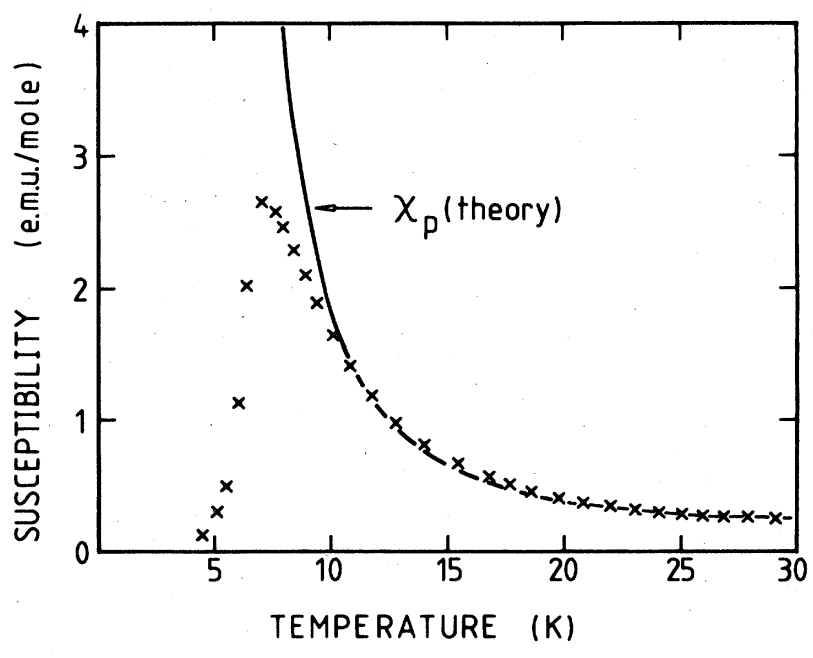

FIG. 1. Magnetic powder susceptibility for pure $\mathrm{FeCl}_{2} \mathrm{py}_{2}$ compared with theory for the ferromagnetic $S=\frac{1}{2}$ Ising chain (solid curve). 
$S=\frac{1}{2}$ Ising chain with Hamiltonian

$$
\begin{aligned}
& \mathscr{H}=-2 J_{z} \sum_{i=1}^{n}\left(S_{i} S_{i+1}+g \mu_{B} \overrightarrow{\mathrm{H}} \cdot \overrightarrow{\mathrm{S}}_{i}\right) ; \\
& \chi_{\|}=\left(N g^{2} \mu_{B}^{2} / 2 J_{z}\right) K \exp (2 K), \\
& \chi_{\perp}=\left(N g^{2} \mu B^{2} / J_{z}\right)\left[\tanh (K)+K \cosh ^{-2}(K)\right],
\end{aligned}
$$

where $K=\left(J_{z} / 2 k_{B} T\right)$ and from which we have calculated the powder susceptibility $\chi_{p}=\left(\chi_{\|}+2 \chi_{\perp}\right) / 3$. The fit yields $J_{z} / k=25 \pm 2 \mathrm{~K}$ with $g=6.6$, the latter value being taken from the saturation moment of $3.3 \mu_{B}{ }^{8}$ In Fig. 2, typical Mössbauer spectra are shown for the $c=0.47 \%$ sample. The entire process of increasing and decreasing $\Gamma$ is now seen to occur at temperatures far above $T_{c} \simeq 3.0 \mathrm{~K}$, since the spectrum is fully hyperfine split at about $5 \mathrm{~K}$, whereas for $T>8 \mathrm{~K}$ the $\Gamma$ of the quadrupole doublet is equal to the instrumental resolution. The temperature dependence of $\Gamma$ deduced from the spectra is plotted in Fig. 3.

In analyzing our results in terms of SG solitons, we start from the classical spin Hamiltonian with isotropic exchange and orthorhombic anisotropy terms:

$$
\mathscr{H}_{\mathrm{FC}}=\sum_{i=1}^{n-1}\left[-2 J \overrightarrow{\mathrm{S}}_{i} \overrightarrow{\mathrm{S}}_{i+1}+A\left(S_{i}^{y}\right)^{2}-D\left(S_{i}^{z}\right)^{2}\right] .
$$

For $J>0, D>0, A>0$, and $D>A$, this would approximately describe the ferromagnetic chain under investigation. Within the continuum approximation, the Hamiltonian (2) can be transformed into its SG form: $:^{3,10}$

$$
\mathscr{H}_{\mathrm{SG}}=E_{0} \int_{-\infty}^{\infty} d x\left(\frac{1}{2} \Psi_{x}^{2}+\frac{1}{2 c_{0}^{2}} \Psi_{t}^{2}+2 m^{2} \sin ^{2} \frac{1}{2} \Psi\right)
$$

Here, $\Psi$ is twice the angle the spins make with the $z$ axis in

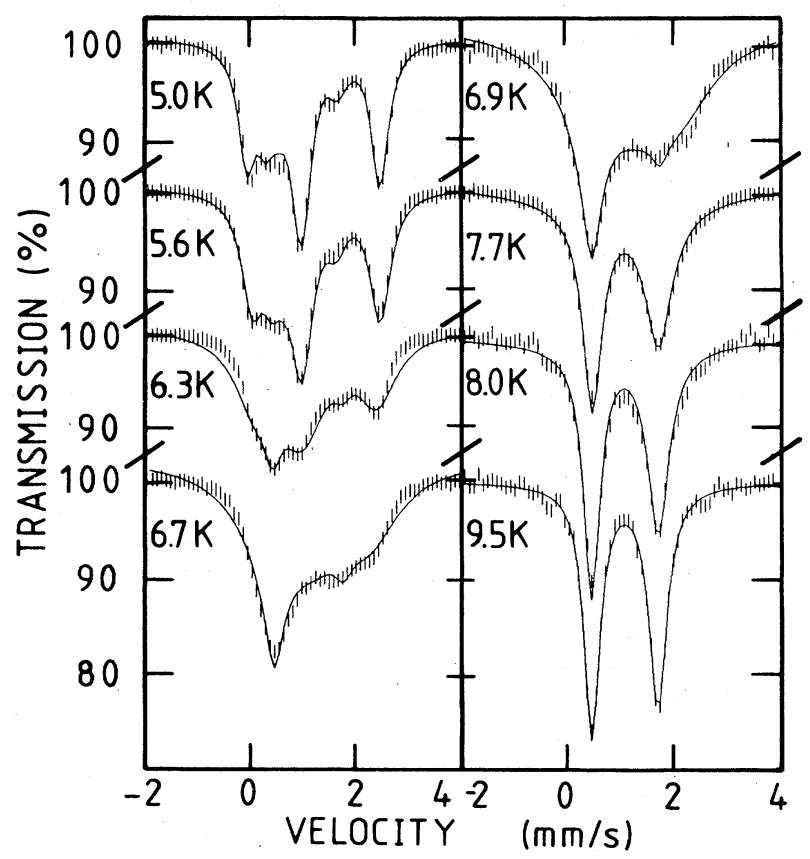

FIG. 2. Mössbauer absorption spectra for the $c=0.47 \% \mathrm{FeCl}_{2} \mathrm{py}_{2}$ sample at different temperatures. Solid lines represent fits to the Blume and Tjon relaxation model.

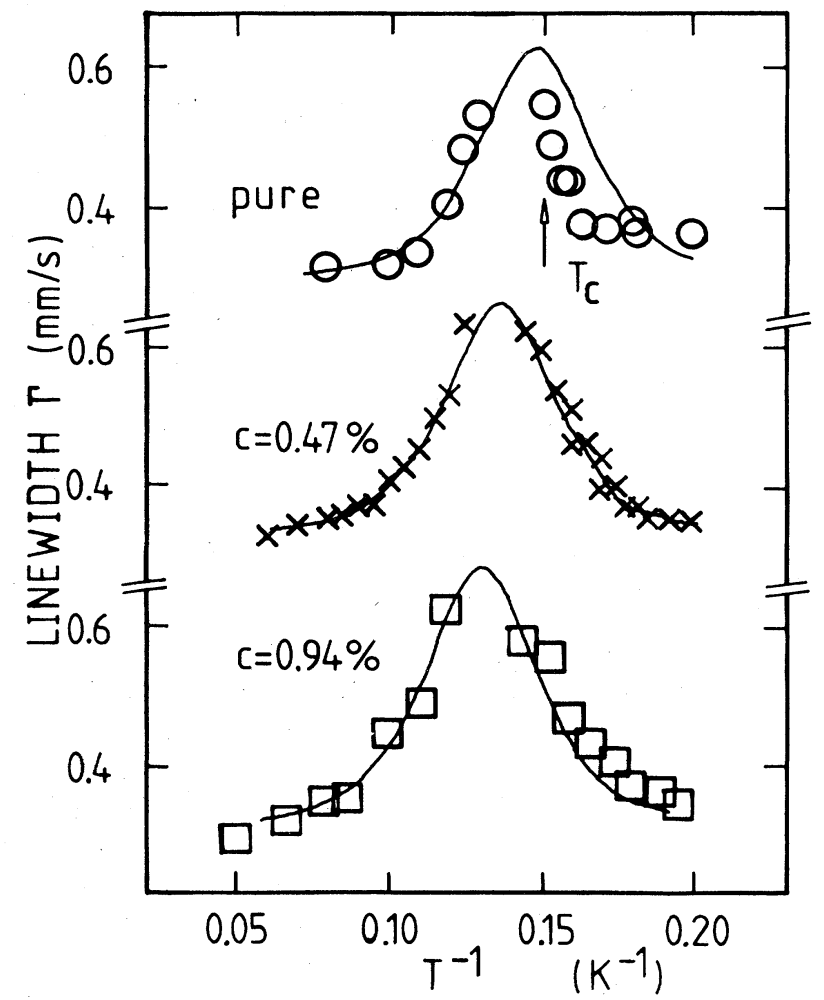

FIG. 3. Experimental linewidths vs inverse temperature. Solid lines are discussed in the text. Spectra were fitted with a superposition of eight Lorentzian lines.

the easy plane. A wall corresponds to a rotation over $180^{\circ}$ of the spin and is a $\pi$ soliton (Bloch wall). The energy scale parameter $E_{0}$, the wall energy $E_{s}$, the maximum wall velocity $c_{0}$, and the soliton rest mass $m_{s}$ are given by $E_{0}=J S^{2} / 2, c^{2}=4 A J S^{2}, E_{s}=(2 D J)^{1 / 2} S^{2}, \quad$ and $m_{s}^{2}=D / J$ respectively. The solitons propagate with mean velocity $v_{s}=c_{0} /\left(4 m_{s} E_{0} / k_{B} T\right)^{1 / 2}$, following the classical thermal distribution. ${ }^{11}$ Since $v_{s}=1 \mathrm{~m} / \mathrm{s}$, the wall passes a lattice site in about $10^{-11} \mathrm{~s}$, i.e., very much shorter than $\omega_{L}^{-1} \simeq 10^{-7} \mathrm{~s}$. The internal structure of the wall will be unimportant, and it is the average time between two passages of a $\pi$ soliton that will determine $\Gamma_{\omega} \cdot 1,2,10,12$ As discussed in Ref. 2, the excess Mössbauer linewidth $\Delta \Gamma$ arising from the spin fluctuations caused by the soliton dynamics will be given by $\Delta \Gamma=A_{\|} S_{\|}\left(k, \omega_{L}\right)$, where $S_{\|}\left(k, \omega_{L}\right)$ is the parallel dynamical structure factor of the Ising system, $\omega_{L}$ the average nuclear Larmor frequency, and $A_{\|}$a constant depending on the hyperfine interaction. As argued by other investigators, ${ }^{3-5}$ one may expect $S_{\|}\left(k, \omega_{L}\right)=\Gamma_{\omega} /\left(\omega_{L}^{2}+\Gamma_{\omega}^{2}\right)$. Here, $\Gamma_{\omega}=4 n_{s} v_{s} / \sqrt{\pi}$ is the flip-rate of the electron spin. For Hamiltonian (3) it is expressed as

$$
\Gamma_{\omega}=\pi^{-1}\left(E_{s} / E_{0}\right) c_{0} \exp \left(-E_{s} / k_{B} T\right) .
$$

As in the previous article the spectra were analyzed assuming Lorentzian line shapes and taking an average $\omega_{L}=10^{7} \mathrm{~Hz}$, which we derived from the hyperfine splitting pattern at low temperatures $(T<5 \mathrm{~K})$. The resulting values for $\Gamma$ are those plotted versus $1 / T$ in Fig. 3 for the three compounds and are compared with results for $\Gamma=\Gamma_{0}+\Delta \Gamma$, where $\Delta \Gamma$ is calculated below. From this figure 
the $3 \mathrm{D}$ ordering and the accompanying sudden decrease of $\Gamma$ at $T_{c}$ for the pure compound is clear. However, in the experimental analysis one is bothered by the fact that the line shape deviates substantially from the Lorentzian approximation when $\Gamma$ goes through its maximum value, and one has the additional complication of four different values of $\omega_{L}$, differing approximately by one order of magnitude. Another problem is the applicability of the classical SG model to strongly anisotropic Ising systems, since the wall width $d_{s}=1 / m s=(J / D)^{1 / 2}$ becomes very small compared to the lattice parameter. Then the continuum approximation loses its validity and the wall can be better approximated by a step function.

In addition to extracting $\Gamma$ from the observed spectra and comparing these with $S_{\|}\left(k, \omega_{L}\right)$, we thus take a different approach and analyze our spectra in terms of the relaxation model of Blume and Tjon. ${ }^{13}$ In this stochastic model, the magnetic hyperfine interaction is replaced by a timedependent hyperfine field $H_{\mathrm{hf}}(t)=H_{0} f(t)$. Applied to our present problem $H_{0}$ is the effective hyperfine field without fluctuations, as derived from the spectra at low temperatures, and the stochastic variable $f(t)$ is a step function which jumps between its maximum and minimum values +1 and -1 . The average time between jumps then corresponds to the flip-rate $\Gamma_{\omega}$. In Fig. 2, the fits to the Mössbauer data, with $\Gamma_{\omega}$ as the only adjustable parameter, are shown. The resulting values for $\Gamma_{\omega}$ are plotted versus $1 / T$ in Fig. 4. Interestingly enough, the two doped compounds show the same exponential dependence within the errors as the pure material.

Although Eq. (4) for $\Gamma_{\omega}$ was derived for SG chains, the exponential term remains valid in the limit of strong Ising anisotropy. For the $S=\frac{1}{2}$ ferromagnetic Ising-type chain this can be shown by applying to the ferromagnetic case the formalism given by Villain, ${ }^{14}$ using the Hamiltonian

$$
\mathscr{H}=-2 \sum_{i=1}^{n-1}\left(J_{z} S_{i} S_{i+1}+J_{x} S_{i} S_{i+1}+J_{y} S_{i} S_{i+1}\right)
$$

to describe the nonlinear excitations in terms of transitions between different magnon bound states. ${ }^{15}$ It can be shown ${ }^{10}$ that for low wall densities $\Gamma_{\omega} \propto \exp \left(-E_{b} / k_{B} T\right)$, where $J_{a}=\left(J_{x}-J_{y}\right) / J_{z}$ and $E_{b} \simeq 2 J_{z}$ is the creation energy of a magnon bound state. Thus, the same exponential term predominates the behavior of the classical SG chain in the continuum limit as well as the $S=\frac{1}{2}$ discrete Ising quantum chain. The analogy between the classical envelope solitons and the magnon bound state has recently been discussed by Schneider. ${ }^{16}$

For the pure sample, 3D ordering occurs near $\Gamma_{\omega}=10^{7} \mathrm{~Hz}$ and the flip-rate slows down quite rapidly because of the progressive blocking of the wall propagation. In the doped

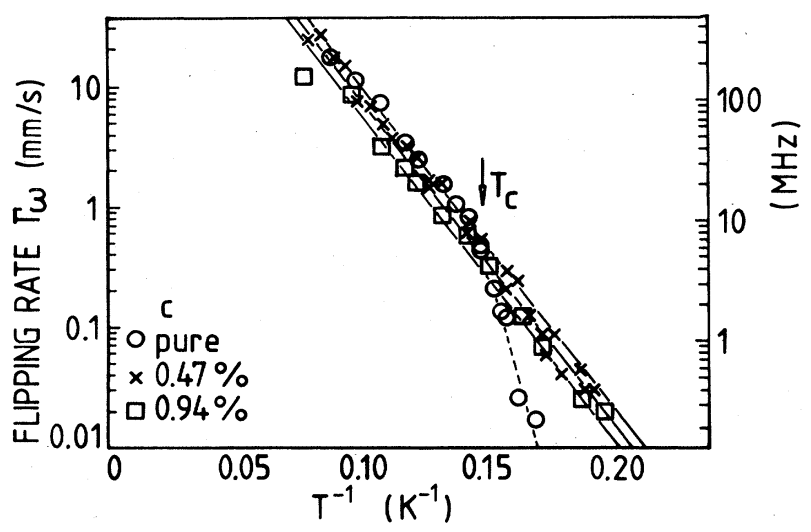

FIG. 4. Electronic flip-rate vs inverse temperature for the three $\mathrm{FeCl}_{2} \mathrm{py}_{2}$ samples. The dashed line is a guide to the eye. The parallel solid curves show the exponential dependences.

samples, 3D ordering is forestalled, and the relaxation rate follows the exponential law to the lowest measurable frequency of the Mössbauer "window" (Fig. 4). The wall energy, given by the slope of the curve, seems to be unaffected by the doping. Evidently the "pure" sample also will contain finite chain segments because of lattice defects, which will typically limit the chain lengths to the order of 500-1000 lattice units. Our results, therefore, show that $E_{s}$ is independent of the chain length for lengths in between $10^{2}-10^{3}$ lattice units. Experimentally we find $E_{s}=60 \pm 2 \mathrm{~K}$ [cf. Eq. (4)]. We may compare this energy with the value for $J_{z} / k_{B}$ from our $\chi$ data, if we take the wall creation energy to be $E_{s} \simeq E_{b} \simeq 2 J_{z}$. Then the Mössbauer experiments yield $J_{z} / k_{B}=30 \pm 1 \mathrm{~K}$, which is close enough to the susceptibility result $J_{z} / k_{B}=25 \pm 2 \mathrm{~K}$.

We also note the shift in the curves in both Figs. 3 and 4 in going from $c=0 \%$ to $c=0.94 \%$. Since $\Gamma_{\omega}=n_{s} v_{s}$, we can interpret this shift as a small reduction of $v_{s}$ caused by the impurities. We note that the microscopic theory of soliton tunneling through impurities is a matter of current theoretical interest. ${ }^{17}$ Lastly we want to emphasize the coherent behavior that we observe in the impure chains, which contrasts with the diffusive solitons reported for impure TMMC $\left\{\left[\left(\mathrm{CH}_{3}\right)_{4} \mathrm{~N}\right]_{2} \mathrm{MnCl}_{4}\right\} .{ }^{18}$ We believe that this is explained by the very small wall width in our Ising-type chains compared to the average chain lengths $\left(10^{2}-10^{3}\right.$ lattice units), combined with the very low wall densities $\left(n_{s}=10^{-5}-10^{-3}\right.$ per lattice unit).

This work is part of the research program of the "Stichting voor Fundamental Onderzoek der Materie," and was made possible by financial support from the "Nederlandse Organisatie voor Zuiver-Wetenschappelijk Onderzoek.",
${ }^{1}$ For a recent brief introduction, see, e.g., L. J. de Jongh, J. Appl. Phys. 53, 8018 (1982), and references therein.

${ }^{2}$ R. C. Thiel, H. de Graaf, and L. J. de Jongh, Phys. Rev. Lett. 47, 1415 (1981).

${ }^{3}$ H. J. Mikeska, J. Phys. C 13, 2913 (1980).

${ }^{4}$ K. Maki, J. Low Temp. Phys. 41, 327 (1981).

5 J. P. Boucher, H. Benner, F. Devreux, L. P. Regnault, J. RossatMignod, C. Dupas, J. P. Renard, J. Bouillot, and W. G. Stirling,
Phys. Rev. Lett. 48, 431 (1982).

6B. F. Little and G. J. Long, Inorg. Chem. 17, 3401 (1978); G. J. Long, D. L. Whitney, and J. E. Kennedy, ibid. 10, 1406 (1971).

${ }^{7}$ P. C. M. Gubbens, W. Ras, A. M. v/dKraan, and J. Reedijk, in Proceedings of the International Conference on Applications of the Mössbauer Effect-1981, Jaipur (The Indian National Science Academy, New Delhi, 1982); the authors have informed us of an error in their temperature calibration. 
${ }^{8}$ S. Foner, R. B. Frankel, E. J. Mcniff, W. M. Reiff, B. F. Little, and G. J. Long, in Magnetism and Magnetic Materials-1974 (San Francisco), edited by C. D. Graham, G. H. Lander, and F. F. Rhyne, AIP Conf. Proc. No. 24 (AIP, New York, 1975), p. 363; S. Foner, R. B. Frankel, W. M. Reiff, B. F. Little, and G. J. Long, Solid State Commun. 16, 159 (1975).

${ }^{9}$ S. Katsura, Phys, Rev. 127, 1508 (1962).

${ }^{10}$ G. Wiersma, H. W. Capel, H. J. M. de Groot, and L. J. de Jongh (unpublished).

11J. F. Currie, J. A. Krumhansl, A. R. Bishop, and S. E. Trullinger,
Phys. Rev. B 22, 477 (1980)

12G. Borsa, Phys. Lett. 80A, 309 (1980).

${ }^{13}$ M. Blume and J. A. Tjon, Phys. Rev. 165, 446 (1968); M. Blume, ibid. 174, 351 (1968).

${ }^{14}$ J. Villain, Physica B 79, 1 (1975); S. E. Nagler, W. J. L. Buyers, R. L. Armstrong, and B. Briat, Phys. Rev. B 28, 387.3 (1983).

15J. B. Torrance and M. Tinkham, Phys. Rev. 187, 587 (1968).

${ }^{16}$ T. Schneider and E. Stoll, J. Appl. Phys. 53, 1850 (1982).

${ }^{17}$ L. Gunther and Y. Imry, Phys. Rev. Lett. 44, 1225 (1980).

${ }^{18}$ Reference 5 ; A. M. C. Tinus, C. J. M. Denissen, H. Nishihara, and W. J. M. de Jonge, J. Phys. C 15, 2791 (1982). 